\title{
Orientações Alimentares na Assistência Pré-natal: Avaliação do processo em Unidades Básicas de Saúde
}

\author{
Food Guidelines in Prenatal Care: Evaluation of the \\ process in Basic Health Units
}

Anna Lícya Calixto Serafim', Amanda de Andrade Marques', Dayanne Braga Cândido', Richelle Moreira Marques'

${ }^{7}$ Centro Universitário de Juazeiro do Norte - UNIJUAZEIRO - Juazeiro do Norte (CE) - Brasil

Email para correspondência: Anna Lícya Calixto Serafim - annalicya@live.com

\begin{abstract}
Resumo
Objetivo: Avaliar as orientações alimentares ofertadas durante o pré-natal em Unidades Básicas de Saúde. Métodos: Trata-se de uma pesquisa de campo de natureza descritiva e de corte transversal com abordagem quantitativa, tendo como lócus 8 Unidades Básicas de Saúde localizadas no município de Caririaçu-CE. A amostra do estudo foi composta por 67 gestantes, tendo como critérios de inclusão: gestantes que realizavam o pré-natal exclusivamente no Sistema Único de Saúde, independentemente da idade, e, mulheres em qualquer período gestacional. Foram excluídas do estudo gestantes que apresentavam alguma deficiência física, mental ou visual e as não alfabetizadas. Como instrumento de coleta de dados foi utilizado um questionário estruturado. Resultados: 0 recebimento de alguma forma de orientação sobre alimentação foi referido por $84 \%$ das gestantes, sendo que apenas $12,4 \%$ foram orientadas somente por nutricionista, enquanto, 42,9\% foram orientadas pelo(a) enfermeiro(a). Quanto ao momento, $80,4 \%$ das participantes relataram que este procedimento foi realizado durante as consultas individuais. Além disso, a recomendação mais abordada às gestantes foi em relação ao aumento da ingestão hídrica, referido por 91\% das participantes. Conclusão: Pode-se verificar através desta pesquisa que as orientações alimentares e nutricionais são realizadas em maior frequência pelo profissional de Enfermagem. Além disso, foi identificado algumas limitações no repasse de orientações especificas a respeito de assuntos voltados para área da Nutrição. Dessa forma, sugere-se a inclusão de uma equipe multidisciplinar para a realização do pré-natal na Atenção Primária e/ou o trabalho integrado com a equipe do Núcleo de Apoio a Saúde da Família.
\end{abstract}

Palavras-chave: Assistência alimentar. Cuidado pré-natal. Gravidez.

\begin{abstract}
Objective: Evaluate the dietary guidelines offered during prenatal care in Basic Health Units. Methods: This is a field research of descriptive and cross-sectional nature with quantitative approach, taking as locus 8 Basic Health Units located in the municipality of Caririaçu-CE. The sample of the study consisted of 67 pregnant women, with inclusion criteria: pregnant women who carry out exclusively on pre-natal Unique Health System, regardless of age, and at any gestational period. Were excluded from the study, pregnant women who had
\end{abstract}


some physical, mental or visual disability and not literate. As data collection instrument was used a structured questionnaire. Results: The receipt of some guidance on food was referred by $84 \%$ of pregnant women, and only $12,4 \%$ were oriented only by the nutritionist while $42,9 \%$ were driven by the nurse. And $80,4 \%$ reported that this procedure was performed during the individual query. In addition, the recommendation more approached at pregnant women was in relation to the increased water intake, referred by $91 \%$ of the participants. Conclusion: It can be seen through this research that the dietary and nutritional guidelines are performed more frequently by the nursing professional. In addition, some limitations were identified in the transfer of specific guidelines regarding issues related to the area of Nutrition. Thus, it is suggested the inclusion of a multidisciplinary team for the performance of prenatal care in Primary Care and / or the integrated work with the team of the Family Health Support Center.

Keywords: Food assistance. Prenatal care. Pregnancy.

\section{INTRODUÇÃO}

A atenção pré-natal tem por finalidade prestar assistência a mulher durante a gravidez, garantindo a evolução da gestação e a manutenção da saúde da mãe e da criança por meio de acompanhamento clínico e educacional, com intuito de reduzir a morbimortalidade materna e infantil. Desse modo, a atenção clínica ofertada durante o período gestacional é fundamental para promover, proteger e recuperar a saúde da gestante e do feto, uma vez que, a gestação é caracterizada por período de intenso crescimento e desenvolvimento do concepto, demandando elevadas necessidades nutricionais decorrentes dos ajustes fisiológicos ${ }^{1-3}$.

A nutrição se faz de grande valia para o prognóstico da gestação pois, as necessidades nutricionais requeridas para esta fase encontram-se aumentadas, provenientes das mudanças ocorridas especificamente para o período gestacional. Sendo assim, o estado nutricional materno antes e durante a gravidez afeta o desenvolvimento do feto, tornando necessário que o profissional de saúde saiba orientar a gestante sobre hábitos alimentares saudáveis e alertá-la para importância desta conduta 4 .

Durante as consultas devem ser realizadas condutas que visem promover o controle das intercorrências gestacionais a fim de minimizar suas complicações e diminuir o índice de mortalidade deste grupo populacional. Porém, foi observado em 2013 a presença de mais de 300 mil mortes maternas no mundo, sendo a maioria em países em desenvolvimento ${ }^{5}$. No Brasil, a morte materna se apresenta como um grave problema de saúde pública e seus elevados percentuais encontram-se, 
principalmente, na região Nordeste. Cabe ressaltar, que os índices mais elevados estão associados a um grupo de mulheres com menor acesso a renda e serviços de saúde qualificados, caracterizando-se como causas evitáveis ${ }^{6}$.

Dessa forma, considera-se importante a realização de avaliações neste serviço para que possa ser possível identificar e apresentar a realidade em que se encontra, no intuito de ofertar um acompanhamento de qualidade que garanta o desenvolvimento da gestação e diminua os índices de mortalidade materna e perinatal observadas, principalmente, através das desigualdades sociais, econômicas, regionais e de acesso aos serviços de saúde7.

Portanto, a ausência de informações apropriadas e adequadas ao período da gravidez, pode ser responsável por um estado nutricional inadequado tendo influência no progresso da gestação ${ }^{8}$. Diante do contexto, questiona-se sobre a situação da assistência nutricional proporcionada pelo Sistema Único de Saúde (SUS) durante as consultas de pré-natal.

Sabe-se que o profissional nutricionista não está inserido na equipe mínima da Estratégia da Saúde da Família (ESF), entretanto, a mesma destaca-se como o principal setor da Atenção Primária a Saúde no Brasil, sendo destinada a prestar assistência contínua por meio das Unidades Básicas de Saúde (UBS) ${ }^{9,10}$. Deste modo, as orientações alimentares e nutricionais são, ou não, realizadas por outros profissionais, que são habilitados para repassar informações de um modo mais amplo e generalista, sendo o profissional nutricionista capacitado para realizar este aconselhamento nutricional. Nesse sentido, acredita-se que ocorra deficiência nas orientações nutricionais ofertadas no pré-natal.

Logo, a temática torna-se relevante a fim de atentar aos Órgãos Públicos sobre a importância da inserção do Nutricionista na equipe mínima da ESF, visto que, este profissional é o capacitado para a realização de prescrição dietoterápica, avaliação e assistência nutricional para os indivíduos.

Neste contexto, a presente pesquisa objetiva avaliar as orientações alimentares ofertadas durante o pré-natal em Unidades Básicas de Saúde do município de Caririaçu-CE. 


\section{METODOLOGIA}

Trata-se de uma pesquisa de campo de natureza descritiva e de corte transversal com abordagem quantitativa, realizada com gestantes em UBS do município de Caririaçu-CE.

Para determinar o número da amostra obteve-se a quantidade de gestantes cadastradas no Sistema de Informação da Atenção Básica (SIAB) do município Caririaçu-CE no primeiro semestre do ano de 2018, sendo indicado 154 gestantes. Dessa forma, para a definição do tamanho da amostra foi realizado um cálculo de amostragem com parâmetro estatístico para margem de erro de $5 \%$ e nível de segurança de $95 \%{ }^{11}$, perfazendo um total de 67 gestantes necessárias para realização da pesquisa.

Dessa forma, foi identificado por meio dos registros da Secretaria da Saúde do Estado do Ceará (2016), o número de Unidades Básicas de Saúde ligadas ao Sistema Único de Saúde, onde apontou-se um total de 18 UBS. Em seguida, realizou-se alocação proporcional, ou seja, foi dividido o número total de gestantes pela quantidade total de UBS para que fosse possível ter um média da quantidade de gestantes atendidas por cada uma, totalizando assim 9 gestantes por UBS. Nesse sentido, para atender a demanda amostral foi necessário consultar 8 UBS, que foram selecionadas por meio de amostragem aleatória simples.

Logo, a amostra do estudo foi composta por 67 gestantes entrevistadas em 8 UBS do município de Caririaçu-CE.

Adotou-se como critérios de inclusão para esta pesquisa: gestantes que realizavam pré-natal exclusivamente no SUS, independentemente da idade, e, mulheres em qualquer período gestacional. Ao mesmo tempo, foram excluídas do estudo gestantes que apresentavam alguma deficiência física, mental ou visual e as não alfabetizadas.

Inicialmente, o projeto da pesquisa foi submetido ao Comitê de Ética e Pesquisa da Faculdade de Juazeiro do Norte, para que se obtivesse autorização para realização da pesquisa. Posterior a aprovação, foi encaminhado um ofício à Secretaria de Saúde do município de Caririaçu-CE, juntamente com o esclarecimento dos objetivos e metodologia proposta pelo referido trabalho, com fins de obter autorização para a realização do mesmo. Mediante a autorização de ambos, foi realizada uma visita em 
cada UBS selecionada, para obter informações sobre o funcionamento da unidade e identificar o melhor dia para aplicação do questionário.

A coleta de dados foi realizada nos meses de setembro e outubro de 2018 , sendo realizada por pesquisadores previamente treinados, utilizou-se como instrumento de coleta um questionário estruturado adaptado.

O questionário foi padronizado contendo questões formuladas a partir das recomendações do Cadernos de atenção básica: atenção ao pré-natal de baixo risco ${ }^{4}$, versando principalmente perguntas referentes a assuntos voltados para área da nutrição que devem ser abordados durante a consulta de pré-natal, mediante o estabelecido pelo Ministério da Saúde. Tais como: uso de ferro e ácido fólico na gestação, importância da suplementação, sintomas causados pela gravidez, ganho de peso repassado pelo profissional de saúde à gestante, interação do ferro com alimentos fontes de cálcio, mudança alimentar na gestação, recebimento de orientações sobre alimentação durante o acompanhamento pré-natal, entre outras. Não foi avaliado o estado nutricional materno, nem foi verificado na Caderneta da Gestante. Como também, não foram investigadas idade, renda e escolaridade. Quanto ao perfil antropométrico das gestantes, o instrumento preconizava identificar apenas se estas tinham sido elucidadas a respeito do seu ganho de peso.

Antes de iniciar a coleta de dados foi realizado um teste piloto em uma UBS não participante do estudo para avaliar se as perguntas elaboradas propagavam dificuldade para o seu entendimento e, observar o tempo necessário para preenchimento do questionário. Com esta avaliação, identificou-se a necessidade de reformular algumas perguntas para uma linguagem mais simples e de fácil compreensão. Posteriormente, iniciou-se as visitas nas UBS nos dias de consulta de pré-natal.

Nesse contexto, as gestantes foram elucidadas sobre a intuito da pesquisa por meio do Termo de Consentimento Livre e Esclarecido (TCLE) sendo que, aquelas que aceitaram participar da pesquisa e assinaram o TCLE, receberam o questionário, juntamente com orientações de como proceder para a resolução das perguntas, para que pudessem respondê-lo. Mediante obtenção dos dados, estes foram expostos em gráficos e tabelas destacando-se as respostas que expuserem maior relevância, em frequências absolutas e relativas, utilizando o programa Microsoft Office Excel 2013. Esta pesquisa atende as normas estabelecidas na Resolução $\mathrm{n}^{\circ} 466$ de 12 de dezembro de 2012 complementada pela Resolução $\mathrm{n}^{\circ}$. 510 de 07 de abril de 2016 do 
Conselho Nacional de Saúde, garantindo a ética da pesquisa por meio do respeito aos participantes, tendo uma prévia autorização livre e esclarecida assegurando sua vontade para participação ${ }^{12,13}$, a mesma recebeu aprovação do Comitê de Ética e Pesquisa da Faculdade de Juazeiro do Norte sob parecer $n^{\circ}: 2.919 .620$, no ano de 2018.

\section{RESULTADOS E DISCUSSÃO}

Quanto à caracterização obstétrica, identificou-se que $76,1 \% \quad(n=51)$ das entrevistadas encontravam-se no $2^{\circ}$ e no $3^{\circ}$ trimestre gestacional; $73 \%(n=19)$ das gestantes que se encontram no $3^{\circ}$ trimestre tiveram seis ou mais consultas de prénatal. Das participantes do estudo $86,6 \%(n=58)$ já haviam apresentado pelo menos uma gestação anterior. Estratificando as gestantes segundo o consumo de suplementação na gestação, 89,6\% $(n=60)$ alegaram fazer uso de suplemento de ferro e 79,1\% (n=53) de ácido fólico, sendo que, 96\% (n=64) tinham conhecimentos dos benefícios desta conduta (Tabela 1).

Tabela 1 - Caracterização das gestantes participantes da pesquisa a respeito do número de consultas de pré-natal, uso de sulfato ferroso e ácido fólico por trimestre gestacional em Unidades Básicas de Saúde do município de Caririaçu-CE, Brasil, 2018

\begin{tabular}{lccc}
\hline Variáveis & $\begin{array}{c}\mathbf{1}^{\circ} \text { Trimestre } \\
\mathbf{n}(\%)\end{array}$ & $\begin{array}{c}\mathbf{2}^{\circ} \text { Trimestre } \\
\mathbf{n}(\%)\end{array}$ & $\begin{array}{c}\mathbf{3}^{\circ} \\
\text { Trimestre } \\
\mathbf{n}(\%)\end{array}$ \\
\hline $\begin{array}{l}\text { Número de gestantes por } \\
\text { trimestre }\end{array}$ & $16(23,9)$ & $25(37,3)$ & $26(38,8)$ \\
Número de Consultas & & & $3(4,5)$ \\
$1-3$ & $12(18)$ & $7(10,5)$ & $13(19)$ \\
$4-6$ & $2(3)$ & $15(22,5)$ & $10(15)$ \\
7 & $2(3)$ & $3(4,5)$ & $25(37,3)$ \\
Está utilizando sulfato & & $19(28,4)$ & $1(1,5)$ \\
ferroso? & & $6(8,9)$ & \\
Sim & $16(23,9)$ & & $19(28,4)$ \\
Não & 0 & $21(31,3)$ & $7(10,4)$ \\
Está utilizando ácido fólico? & $13(19,4)$ & $4(6)$ & \\
Sim & $3(4,5)$ & & \\
Não & &
\end{tabular}

n- número de gestantes

O recebimento de alguma forma de orientação sobre alimentação durante o acompanhamento de pré-natal foi referido por $84 \%(n=56)$ das gestantes, das quais, $42,9 \%(n=24)$ afirmaram que quem orientou foi o(a) enfermeiro(a) seguido por $23,2 \%(n=13)$ o(a) médico(a) e o(a) enfermeiro(a) e apenas $14,2 \%(n=8)$ foram orientadas somente pelo(a) nutricionista. Sobre o local de realização das orientações, $80,4 \%(n=45)$ informaram ter ocorrido nas consultas individuais. Os 
resultados sobre a caracterização das orientações alimentares recebidas durante o acompanhamento de pré-natal estão descritos na (Tabela 2).

Tabela 2 - Caracterização das orientações nutricionais recebidas durante o acompanhamento pré-natal em Unidades Básicas de Saúde do município de Caririaçu-CE, Brasil, 2018

\begin{tabular}{lcc}
\hline Variável & $\mathbf{n}$ & $\%$ \\
\hline Recebimento de orientação sobre & & \\
alimentação & 56 & 84 \\
Sim & 11 & 16 \\
Não & 67 & 100 \\
Total & & \\
Profissional que orientou & 24 & 42,9 \\
Enfermeiro(a) & 13 & 23,2 \\
Enfermeiro(a) e médico(a) & 8 & 14,2 \\
Nutricionista & 7 & 12,5 \\
Médico(a) & 2 & 3,6 \\
Nutricionista e enfermeiro(a) & 2 & 3,6 \\
Nutricionista, enfermeiro(a) e médico(a) & 0 & 0 \\
Outro & 56 & 100 \\
Total & & \\
Local da orientação & 45 & 80,4 \\
Consulta & 4 & 7 \\
Palestra & 3 & 5,4 \\
Grupo de gestante & 2 & 3,6 \\
Consulta e palestra & 2 & 0 \\
Palestra e grupo de gestante & 0 & 100 \\
Outro local & 56 & \\
Total & &
\end{tabular}

n- número de gestantes

Na figura 1 está exposta a caracterização do conteúdo das orientações nutricionais que devem ser abordadas no pré-natal. Logo, as participantes da pesquisa foram questionadas se receberam, ou não, tais recomendações. Foi perceptível que a recomendação mais repassada às gestantes foi em relação ao aumento da ingestão hídrica, onde 91\% ( $n=61)$ afirmaram receber esta informação, seguida pelo aumento do consumo de frutas e legumes (88\%) e redução no consumo de sal (88\%).

Figura 1 - Orientações alimentares repassadas durante a consulta de pré-natal em Unidades Básicas de Saúde do município de CaririaçuCE, Brasil, 2018

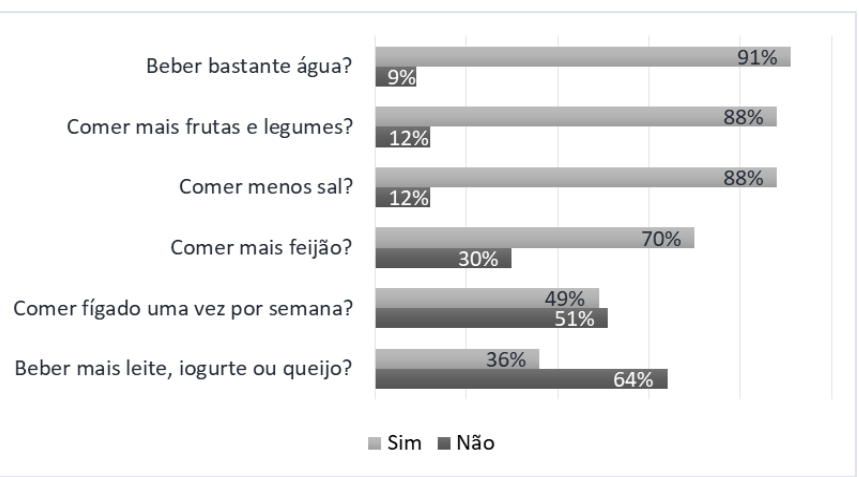


No que se refere aos alimentos fontes de ferro, a figura 2 exibe a verificação de recomendações quanto a ingestão deste nutriente, visto que, sua biodisponibilidade pode ser afetada por outros alimentos.

Figura 2 - Verificação do repasse de orientações para o consumo de alimentos fontes de ferro em Unidades Básicas de Saúde do município de Caririaçu-CE, Brasil, 2018.

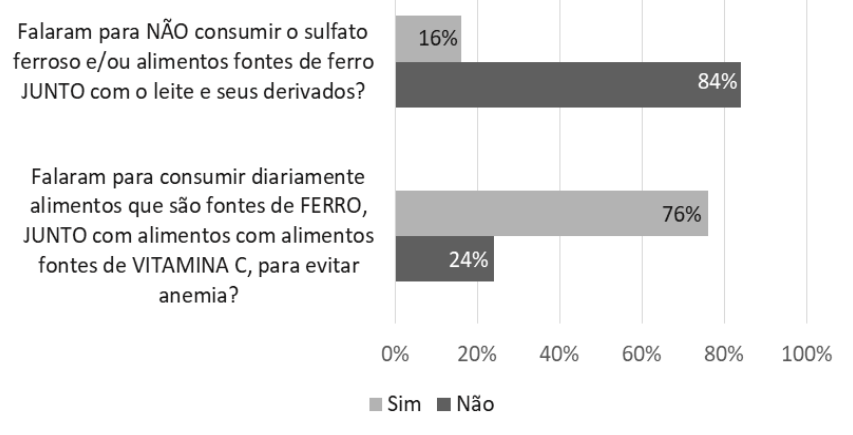

Em relação a sintomas apresentados na gravidez, os mais citados foram enjoo $71 \%$ $(n=48)$ e azia $61 \%(n=41)$. Questionadas a respeito da ajuda do pré-natal para repassar orientações que ajudasse a amenizar os sintomas, 52,2\% $(n=35)$ das participantes declararam que a ajuda foi eficiente.

Além disso, no que tange ao perfil antropométrico das gestantes, 56,7\% ( $n=38)$ apontaram ganho de peso normal, 19,4\% ( $n=13)$ expuseram estar com ganho elevado, $13,4 \%(n=9)$ alegaram estar ganhando pouco peso e 10,4\% (n=7) apontaram não ter conhecimento do seu ganho de peso durante a gestação.

\section{DISCUSSÃO}

Em relação ao número de consultas durante a gestação, 73\% (n=19) das gestantes que se encontram no $3^{\circ}$ trimestre tiveram seis ou mais consultas de pré-natal, até o momento de realização deste estudo. Esta quantidade de consultas atende as recomendações do Ministério da Saúde ${ }^{4}$, que preconiza no mínimo 6 consultas de pré-natal para gestantes de baixo risco. Além disso, em outra pesquisa brasileira ${ }^{6}$ foi constatado que a região Nordeste foi a única com cobertura de acompanhamento acima da média nacional. Verificou-se também, que as gestantes que realizaram menos de sete consultas durante o pré-natal apresentam maiores chances de gerar bebês prematuros, com baixo peso ao nascer e/ou de óbito perinatal14.

As questões sobre alimentação expostas na figura 1, tratam-se de orientações alimentares preconizadas pelo Ministério da Saúde ${ }^{4}$ para serem repassadas durante 
a consulta pré-natal visando garantir o estado nutricional adequado e o desenvolvimento da gestação.

Enfatizando as orientações alimentares durante o pré-natal, 84\% (n=56) das entrevistadas receberam alguma orientação sobre alimentação, sendo que, 80,4\% $(n=45)$ foi orientada durante sua consulta, apresentando similaridade com outro estudo publicado na literatura científica ${ }^{15}$. Além disso, constatou-se que o profissional enfermeiro(a) foi o que repassou as informações nutricionais com maior frequência ao público alvo, em relação ao profissional nutricionista e médico(a), episódio evidenciado também em outro estudo ${ }^{16}$.

Por meio da pesquisa foi possível identificar que as orientações nutricionais repassadas com maior frequência se tratam de informações gerais sobre alimentação como: beber bastante água, diminuir o consumo de sal e aumentar o consumo de frutas e verduras. No entanto, é essencial que orientações alimentares e nutricionais mais específicas para o período gestacional sejam abordadas durante o pré-natal. Logo, averígua-se a necessidade de um profissional Nutricionista para fazer parte deste acompanhamento, esteja ele inserido na ESF ou no Núcleo de Apoio à Saúde da Família (NASF).

Orientação como interação de alimentos fontes de ferro ou do sulfato ferroso com leite e seus derivados, apesar de não estar presente nas recomendações de pré-natal pelo Caderno de Atenção Básica, é importante ser repassada para as gestantes pois, esta interação poderá afetar a biodisponibilidade do nutriente e no período gestacional a mulher demanda de um aporte maior de ferro ${ }^{17}$. No entanto, foram reportadas por apenas $16 \%(n=11)$ das participantes.

Foi demonstrado em um estudo brasileiro, que apenas 53,3\% das gestantes participantes declararam realizar o consumo diário de leite e derivados ${ }^{18}$. Associado a este fato, em outra pesquisa que apresentava como objetivo identificar a prevalência de inadequação da ingestão, de cálcio e vitamina $\mathrm{D}$, em gestantes atendidas no pré-natal de baixo risco da rede pública de atenção primária à saúde evidenciou-se uma inadequação maior que 70\% em relação ao consumo diário de cálcio, variando para mais ou menos conforme o trimestre gestacional ${ }^{19}$. Nesta pesquisa, menos da metade das gestantes afirmaram terem sido aconselhadas a aumentar o consumo de leite e derivados.

Logo, pode-se inferir que as gestantes estão sendo pouco orientadas no atendimento pré-natal para realizarem o consumo de três porções diárias de leite e derivados. E o 
repasse desta informação é importante para evitar deficiência de cálcio que pode refletir em problemas ósseos e dentários para mãe e para o feto ${ }^{20}$.

Referente ao uso da suplementação de ferro e ácido fólico, foi descrito por mais da metade das gestantes a sua utilização, resultado similar ao encontrado em outra pesquisa $^{21}$. A deficiência destes nutrientes pode acarretar em defeitos no fechamento do tubo neural, baixo peso ao nascer, maior risco de infecção, prematuridade e déficit cognitivo no recém-nascido ${ }^{22}$.

Quanto ao perfil antropométrico das gestantes, 56,6\% $(n=38)$ afirmaram estar com peso adequado. Dentro deste contexto, cabe ressaltar que o estado nutricional materno é um indicador importante para o prognóstico da gravidez, em virtude dos riscos que a inadequação do mesmo pode gerar ao binômio, sendo assim, a equipe de saúde tem papel fundamental na prevenção e identificação das gestantes de risco e o índice de massa corporal é um dos determinantes para a gestação saudável, pois quando adequado, diminui o risco de intercorrências ${ }^{23-24}$.

Nesse sentido, é sabido que o estado nutricional inadequado durante a gravidez pode acarretar em consequências danosas tanto para mãe quanto para o feto. O ganho de peso acima do recomendado pode acarretar em Síndrome Hipertensiva Gestacional, Diabetes Mellitus Gestacional, macrossomia, sofrimento fetal e trabalho de parto prolongado, enquanto o baixo peso pode gerar parto prematuro, baixo peso no recém-nascido e aumento das taxas de morbimortalidade perinatal ${ }^{25}$.

Em um estudo que objetivava descrever as percepções maternas sobre a assistência nutricional fornecida através do acompanhamento interdisciplinar do pré-natal e puerpério em uma Unidade Básica de Saúde, o autor constatou por meio dos relatos das mães a eficácia da atuação de uma equipe multidisciplinar no pré-natal, tendo em vista, a satisfação apresentada pelas participantes do estudo e a importância da assistência nutricional para a saúde materno-infantil, evidenciou-se também elevação da qualidade do serviço ofertado ${ }^{26}$.

Nesse contexto, os autores de duas pesquisas brasileiras ${ }^{27,28}$, que visaram avaliar o perfil nutricional de gestantes e avaliar a atenção nutricional de gestantes de baixo risco, concluíram que a atenção multiprofissional ofertada, em especial, pela Atenção Primária durante o pré-natal deve adotar maior estima para atenção nutricional oferecida dentro deste setor visto sua contribuição para o desfecho da gestação. 
Este estudo, em particular, apresenta limitação no instrumento de coleta, pois o mesmo não coletou dados sobre a idade das participantes, o ganho de peso foi apenas relatado e o instrumento poderia ter abrangido mais questões voltadas para a alimentação das gestantes, todavia, os objetivos da pesquisa foram alcançados.

\section{CONCLUSÃO}

Pode-se verificar através desta pesquisa que as orientações alimentares e nutricionais ofertadas pela Estratégia de Saúde da Família na percepção das gestantes foi realizada em maior frequência pelo profissional de Enfermagem.

Logo, foi identificado algumas limitações no repasse de orientações especificas a respeito de assuntos voltados para área da Nutrição que competem ao Nutricionista abordar, fato este que reforça a importância da atenção integral no atendimento à gestante.

Nesse contexto, a participação do profissional Nutricionista é fundamental para o desfecho da gestação, visto que, a Nutrição contribui para o estado nutricional e o ganho de peso adequados neste período, amenização de sintomas provocados pela gestação como: náuseas, vômitos, azia e constipação; prevenção de doenças como: diabetes, hipertensão e anemia.

Dessa forma, sugere-se a inclusão de uma equipe multidisciplinar para a realização do pré-natal na Atenção Primária e/ou o trabalho integrado com a equipe do Núcleo de Apoio a Saúde da Família, tendo em vista que esta equipe conta a presença do Nutricionista e tem como papel apoiar a ESF por meio do compartilhamento de conhecimento visando melhorar o atendimento de saúde ofertado a população.

\section{REFERÊNCIAS}

1. Tourinho AB, Reis LBSM. Peso ao Nascer: uma abordagem nutricional. Com. Ciências Saúde. 2013; 22 (4): 19-30.

2. Andrade BD, Silva ACP, Santos MTM, Campos T, Luquetti SCPD, Cândido APC, et al. Fatores nutricionais e sociais de importância para o resultado da gestação, em mulheres em acompanhamento na rede de atenção primária de Juiz de Fora. Rev Med Minas Gerais. 2015; 25 (3): 344-52. DOI: 10.5935/2238-3182.20150069.

3. Guimarães WSG, Parente RCP, Guimarães TLF, Garnelo L. Acesso e qualidade da atenção pré-natal na Estratégia Saúde da Família: infraestrutura, cuidado e gestão. Cad. Saúde Pública. 2018; 34 (5): 1-13. DOI: 10.1590/0102-311X00110417.

4. Ministério da Saúde (BR). Secretaria de Atenção à Saúde. Departamento de Atenção Básica. Cadernos de atenção básica: atenção ao pré-natal de baixo risco. $1^{\mathrm{a}}$ ed. Brasília (DF); 2013. 
5. Kassebaum NJ, Bertozzi-Villa A, Coggeshal MS, Shackelford KA, Steiner C, Heuton KR, et al. Global, regional, and national levels and causes of maternal mortality during 1990-2013: a systematic analysis for the Global Burden of Disease Study 2013. The Lancet 2014; 384 (9947): 980-1004. DOI: 10.1016/ S0140-6736(14)60696-6.

6. Nogueira CMCS, Justino JMR, Tavares MIPL, Morais FRR. Caracterização da infraestrutura e do processo de trabalho na assistência ao pré-natal. Rev Cogitare Enferm. 2016; 21 (4): 01-10.

7. Nunes JT, Gomes KRO, Rodrigues MTP, Mascarenhas MDM. Qualidade da assistência pré-natal no Brasil: revisão de artigos publicados de 2005 a 2015. Cad. Saúde Colet. 2016; 24 (2): 252-61. DOI: 10.1590/1414-462X201600020171.

8. Abreu LG. Avaliação do estado nutricional das gestantes acompanhadas em uma equipe de estratégia saúde de família na cidade de Montes Claros - MG [trabalho de conclusão de curso]. Montes Claros (MG): Universidade Federal do Triângulo Mineiro; 2016.

9. Niquini RP. Avaliação da assistência nutricional no pré-natal em sete unidades de saúde da família do município do Rio de Janeiro [dissertação de mestrado]. Rio de Janeiro (RJ): Escola Nacional de Saúde Pública Sergio Arouca / ENSP; 2010.

10. Ministério da Saúde (BR). Política Nacional de Atenção Básica. $1^{\mathrm{a}}$ ed. Brasília (DF); 2012.

11. Bussab WO, Morettin PA. Estatística Básica 8a ed. São Paulo (SP): Saraiva; 2013.

12. Ministério da Saúde (BR). Conselho Nacional de Saúde. Resolução CNS nº 466/12 de 12 de dezembro de 2012. Brasília, $1^{\mathrm{a}}$ seção: 1-12, 2012.

13. Ministério da Saúde (BR). Conselho Nacional de Saúde. Resolução CNS no 510/16 de 07 de abril de 2016. Brasília, 1 ${ }^{\mathrm{a}}$ seção: 44-6, 2016.

14. Nascimento IB, Pacheco VC, Souza MLR, Pinheiro EB, Silva TR, Fleig R et al. Assistência pré-natal e resultado perinatal. Rev. Bras Promoç Saúde. 2017; 30 (2): 187-94. DOI: 10.5020/18061230.2017.

15. Lisboa CS, Bittencourt LJ, Santana JM, Santos DB. Assistência nutricional no pré-natal de mulheres atendidas em unidades de saúde da família de um município do Recôncavo da Bahia: um estudo de coorte. Rev. Demetra. 2017; 12 (3): 713-73. DOI: 10.12957/demetra.2017.28439.

16. Matos MR, Condas BA, Cappelletti C, Skupien SV. Atuação do profissional enfermeiro no pré-natal: educando para saúde. Artigo apresentado no XIII Congresso Nacional de Educação; 2017 ago. 28-31; Curitiba (PR), Brasil.

17. Calheiros MSC. Efetividade do programa nacional de suplementação de ferro: estudo de base populacional com lactentes 6 a 18 meses do estado de Alagoas. [dissertação de mestrado]. Maceió (AL): Universidade Federal de Alagoas / UFA; 2017.

18. Ferreira RC, Bezerra AR, Tavares MCM, Tenório MCS, Barros AMR, Oliveira ACM. Padrão de consumo dietético de gestantes e sua relação com a insegurança alimentar no domicílio. BRASPEN J. 2017; 32 (2): 128-33.

19. Gomes CB, Malta MB, Corrente JE, Benício MHA, Carvalhaes MABL. Alta prevalência de inadequação da ingestão dietética de cálcio e vitamina $\mathrm{D}$ em duas coortes de gestantes. Cad. Saúde Pública. 2016; 32 (12): 1-12. DOI: 10.1590/0102311X00127815. 
20. Premaor MO, Brondani JE. Nutrição e saúde óssea: a importância do cálcio, fósforo, magnésio e proteínas. Rev. da AMRIGS. 2016; 60 (3): 253-63.

21. Murakami PY, Höfelmann DA. Uso de suplementos de ácido fólico e ferro em gestantes de uma unidade de saúde do Paraná. Rev. Bras. Pesq. Saúde. 2016; 18 (3): 100-13.

22. Medeiros RRS, Nóbrega MM, Santos MLL, Vieira TG. Percepção de gestantes acerca da importância do uso do ácido fólico e sulfato ferroso e o papel assistencial da enfermagem na atenção primária. Rev Temas em Saúde. 2016; 16 (4): 295-310.

23. Gomes RNS, Gomes VTS, Caldas DRC, Lago EC, Campos FKL, Gomes MS. Avaliação do estado nutricional de gestantes atendidas em unidades básicas de saúde de Caxias/Ma. Rev Interd. 2014; 7 (4): 81-90.

24. Fonseca MRCC, Laurenti R, Marin CR, Traldi MC. Ganho de peso gestacional e peso ao nascer do concepto: estudo transversal na região de Jundiaí, São Paulo, Brasil. Rev Ciência \& Saúde Coletiva. 2014; 19 (5): 1401-07. DOI: 10.1590/141381232014195.17022013.

25. Teixeira CSS, Cabral ACV. Avaliação nutricional de gestantes sob acompanhamento em serviços de pré-natal distintos: a região metropolitana e o ambiente rural. Rev. Bras Ginec Obst. 2016; 38 (1): 27-34. DOI: http://dx.doi.org/10.1055/s-0035-1570111.

26. Barbosa AM, Araújo LSA, Barbosa VMO, Guerra FAM. Percepções maternas sobre a assistência nutricional no acompanhamento interdisciplinar do pré-natal e puerpério. Rev Tempus Actas de Saúde Coletiva. 2018; 11 (2): 09-24. DOI: http://dx.doi.org/10.18569/tempus.v10i4.2047.

27. Moreira MA, Lessa SS, Carrasco MG, LO, Silva SS, Santiago HO. Perfil Nutricional De Gestantes Acompanhadas em uma Unidade De Saúde Da Família. Rev Saúde e Desenvolvimento. 2015; 8 (4): 159-73.

28. Soares LA, Lima DB. Atenção Nutricional Às Gestantes De Baixo Risco: Contribuições Para As Políticas Públicas. Rev Saúde e Pesquisa. 2018; 11 (2): 38594. DOI: http://dx.doi.org/10.177651/1983-1870.2018v11n2p385-394. 